\title{
A Nonlinear Frequency-Domain Approach for Numerical Simulation of Wave Energy Converters
}

\author{
Alexis Mérigaud ${ }^{(}$and John V. Ringwood, Senior Member, IEEE
}

\begin{abstract}
Nonlinear, analytical wave-energy converter (WEC) models are generally simulated through time-domain (TD) numerical integration. However, the relatively high computational requirements of TD integration are not compatible with applications where a large number of simulations are needed. Spectral domain (SD) linearization has also been proposed to take into account some nonlinear effects, while being much faster than TD integration. However, as explained in this paper, such SD models have limited accuracy, and cannot extend to the static nonlinear forces. In this paper, a nonlinear frequency-domain (NLFD) method is investigated for WEC simulation, using a projection of the dynamical equations onto a basis of trigonometric functions. A comparison with TD integration (second-order Runge-Kutta-RK2) and SD methods is provided, through theoretical considerations, and by means of numerical simulations based on two case studies. In the cases considered, the proposed NLFD method allows for significant computational savings compared to RK2 integration, without requiring any approximation for the radiation forces. NLFD thus shows promising potential for applications involving extensive WEC simulation, such as power production assessment, while preserving the WEC model accuracy. Although slower than SD, NLFD has significant benefits in terms of accuracy and range of applicability.
\end{abstract}

Index Terms-Frequency domain, galerkin method, nonlinearities, numerical simulation, spectral domain, time domain, wave energy converters.

\section{INTRODUCTION}

A NALYTICAL WEC models, based on linear potential flow theory, are commonplace in the wave energy field [1], and are needed for a variety of purposes, such as power production assessment, parametric optimisation and probabilistic studies. The common characteristic of these applications is the requirement for a large number of simulations, which must be carried out within a reasonable amount of time, and can be achieved only if the WEC response in each sea state is derived through efficient numerical techniques.

Provided that the WEC considered can be modelled linearly with good accuracy, linear frequency-domain models constitute the most traditional example of such computationally-efficient

Manuscript received December 12, 2016; revised April 13, 2017; accepted June 4, 2017. Date of publication June 16, 2017; date of current version December 14, 2017. This work was supported by the Science Foundation Ireland under Grant 12/RC/2302 for the Marine Renewable Ireland (MaREI) Centre. Paper no. TSTE-00983-2016. (Corresponding author: Alexis Mérigaud.)

The authors are with the Centre for Ocean Energy Research, Department of Electronic Engineering, Maynooth University, Maynooth, Ireland (e-mail alexis.merigaud.2015@mumail.ie; john.ringwood@eeng.nuim.ie).

Color versions of one or more of the figures in this paper are available online at http://ieeexplore.ieee.org.

Digital Object Identifier 10.1109/TSTE.2017.2716826 methods [2]; knowing the frequency-domain representation of the incident waves, and the linear frequency-domain transfer function that relates input waves to any output of interest (WEC position, velocity, etc.), a simple multiplication of the input wave representation by the transfer function is sufficient to derive the required output.

However, the dynamics of many WECs are affected by various sources of non-linearity, such as hydrodynamic effects including non-linear Froude-Krylov forces and viscous drag terms, or non-linear characteristics in the moorings, in the power take-off (PTO) system, or in the WEC control strategy. When such non-linear effects play a significant role in the WEC dynamics, the classical, linear frequency-domain formulation is inadequate.

The most obvious way to take into account non-linear effects in the WEC response consists of carrying out simulations in the time domain (TD) [1], whereby the dynamical equations are integrated through a numerical procedure. TD simulations can estimate the WEC response, irrespective of the form of the non-linear forces and of the input waves. They are therefore, when non-linearities are present, a suitable tool for the detailed study of the WEC time-domain dynamics, and for the study of transient events in particular. However, the accuracy and versatility of numerical integration can be at the expense of computational efficiency; in particular, the calculation of linear radiation forces through a convolution product [3] represents a major computational burden, which can only be circumvented through radiation force approximation methods [4].

Spectral-domain (SD) linearisation has been suggested as a possible alternative to include some types of nonlinear effects while keeping computational requirements low [1]. SD linearisation is based on a statistical representation of the WEC inputs and outputs as stationary Gaussian processes.

In this paper, a non-linear frequency-domain (NLFD) simulation method is proposed, using a projection of the system inputs and outputs onto a basis of trigonometric polynomials. Using harmonic balance, the dynamical equations take the form of a set of non-linear equations, which can be solved using a gradient-based technique. The proposed approach, which is a particular form of Galerkin method, has been used in [5] and [6] for vibration analysis of offshore structures with relatively weak non-linearities.

A frequency-domain representation of WECs subject to nonlinearities has been used before, within the scope of WEC power-maximising control, for example in [7] and other works by the same author; the frequency-domain formalism allows for 
expressing the cost to minimise as a function of a limited number of variables (i.e. the frequency-domain coefficients for position, velocity and control forces). The dynamical equations are considered as equality constraints, which must hold at a set of "collocation points" in the time-domain. In contrast, the technique presented here solves a simulation problem - not a control problem - and represents the non-linear dynamics in the frequency domain, as a harmonic balance equation. Another main distinctive feature of the NLFD technique is the use of an explicit Jacobian computation of the terms constituting the nonlinear, frequency-domain dynamical equation, which allows for a fast convergence of the algorithm.

To the authors' knowledge, this study is the first application of the technique for WEC simulation. For the sake of simplicity, the NLFD method will be presented here with one degree of freedom (DoF) only, but application to systems with multiple DoFs is possible [6]. Further, a comparison with TD and SD simulation techniques is provided. Using $2^{\text {nd }}$-order Runge-Kutta (RK2) integration [8], with different time steps, and with direct radiation force computation, allows for validating the accuracy of the NLFD method, and tends to show that the computational savings of NLFD, with respect to TD simulation with direct radiation force calculation, are at least comparable to the gain resulting from radiation force approximation techniques. The method also overcomes most of the limitations inherent to SD in terms of range of applicability and accuracy.

The NLFD method is briefly introduced in Section II. Section III details how NLFD relates to TD and SD methods, explaining, in particular, why NLFD is not subject to the same theoretical limitations as SD. To serve as a basis for numerical comparison of TD, SD and NLFD, two non-linear WEC models are described in Section IV. The corresponding numerical results are presented in Section V. Finally, Section VI discusses the benefits and limitations of the proposed NLFD method, and highlights possible directions for future research.

\section{Presentation of the NLFD Method}

\section{A. Non-Linear Frequency-Domain Formulation}

Let us consider the motions of a one DoF WEC. The WEC displacement and its derivatives are denoted, respectively, $\zeta$, $\dot{\zeta}$ and $\ddot{\zeta}$. Assuming that the WEC dynamics can be linearly described, the time-domain equations of motion are given by the well-known Cummins equation [3]:

$$
\left(\mu+\mu_{\infty}\right) \ddot{\zeta}+\int_{-\infty}^{t} k_{\text {rad }}(t-\tau) \dot{\zeta}(\tau) d \tau+k_{h} \zeta-f_{e}-f_{P T O}=0
$$

where

1) $\mu$ is the WEC inertia;

2) $k_{h}$ is the hydrostatic stiffness coefficient;

3) the radiation forces are computed as the sum of an inertial term $\mu_{\infty} \ddot{\zeta}$ and a convolution product between the past values of the velocity and the radiation impulse response function $k_{\text {rad }}$;

4) $f_{e}$ is the wave excitation force, and $f_{P T O}$ is the force exerted on the device by the PTO system.
Let us write a nonlinear extension or modification of (1), where some of the forces are now described as non-linear functions of the WEC displacement and velocity. It is convenient to write (1) in the form

$$
g_{l}(\zeta, \dot{\zeta}, \ddot{\zeta})-f_{n l}(\zeta, \dot{\zeta}, t)-e(t)=0
$$

where $g_{l}$ contains the inertial terms and linear forces, $f_{n l}$ contains the forces that are modelled non-linearly, and $e$ represents an additive input, e.g. linear excitation forces or diffraction forces. Such a formulation applies to a wide class of WEC models, and allows for the inclusion of various non-linear forces such as non-linear restoring or Froude-Krylov forces, viscous drag terms, mooring line effects, non-linear PTO force, etc. Of course, the hydrodynamic force terms that are non-linearly modelled in $f_{n l}$ must be accordingly removed from $g_{l}$.

Let us now consider the steady-state motion of a WEC, subject to a periodic input wave signal $\eta$, described as a finite sum of harmonic sinusoids:

$$
\eta(t) \approx \sum_{k=1}^{N} a_{\eta_{k}} \cos \left(\omega_{k} t\right)+b_{\eta_{k}} \sin \left(\omega_{k} t\right)
$$

where $\forall k \in[1 ; N], \omega_{k}=k \omega_{0}, \omega_{0}=\frac{2 \pi}{T}, T$ is the time period after which $\eta$ is repeated, and the component amplitudes $a_{k}$ and $b_{k}$ are derived from the wave spectrum. Furthermore, since only the WEC steady-state regime is considered, all the system variables are also assumed to be well approximated through truncated sums of sinusoids, so that:

$$
\begin{aligned}
& \zeta(t) \approx a_{\zeta_{0}}+\sum_{k=1}^{N} a_{\zeta_{k}} \cos \left(\omega_{k} t\right)+b_{\zeta_{k}} \sin \left(\omega_{k} t\right) \\
& e(t) \approx a_{e 0}+\sum_{k=1}^{N} a_{e k} \cos \left(\omega_{k} t\right)+b_{e k} \sin \left(\omega_{k} t\right)
\end{aligned}
$$

Finally, denoting $X=\left(a_{\zeta_{1}}, \ldots, a_{\zeta_{N}}, b_{\zeta_{1}}, \ldots b_{\zeta_{N}}, a_{\zeta_{0}}\right)^{\mathrm{T}}$ and $E=\left(a_{e 1}, \ldots, a_{e N}, b_{e 1}, \ldots b_{e N}, a_{e 0}\right)^{\mathrm{T}}$, (2) can be written in the form of a non-linear harmonic balance equation:

$$
M X-F(X)-E=0_{\mathbf{R}^{2 N+1}},
$$

where

1) The terms of $g_{l}$ are transcribed into matrix form as $G_{l}(X)=M X$. In particular, using Ogilvie's relation [9], the radiation terms simplify into the frequency-dependent radiation added mass and damping $A_{\text {rad }}(\omega)$ and $B_{\text {rad }}(\omega)$, as shown in [7]. Typically, when both radiation and hydrostatic restoring forces are linearly modelled, the components of $M$ at row $i$ and column $j$ are, $\forall i, j \in[1 \ldots N]^{2}$,

$$
\begin{gathered}
M_{i, j}= \begin{cases}-\omega_{i}^{2}\left(\mu+A_{\text {rad }}\left(\omega_{i}\right)\right)+k_{h}, & i=j \\
0, & i \neq j\end{cases} \\
M_{i+N, j+N}=M_{i, j} \\
M_{i, j+N}= \begin{cases}\omega_{i} B_{\text {rad }}\left(\omega_{i}\right), & i=j \\
0, & i \neq j\end{cases} \\
M_{i+N, j}=-M_{i, j+N} \\
\text { and } M_{2 N+1,2 N+1}=k_{h}
\end{gathered}
$$


2) $F(X)$ represents the projection, similarly to (3), of the non-linear forces $f_{n l}$, so that $\forall i \in[1 \ldots N]$ :

$$
\begin{aligned}
F_{i}(X) & =\frac{2}{T} \int_{0}^{T} f_{n l}\left(\zeta_{X}, \dot{\zeta}_{X}, t\right) \cos \left(\omega_{i} t\right) d t \\
F_{i+N}(X) & =\frac{2}{T} \int_{0}^{T} f_{n l}\left(\zeta_{X}, \dot{\zeta}_{X}, t\right) \sin \left(\omega_{i} t\right) d t
\end{aligned}
$$

and

$$
F_{2 N+1}(X)=\frac{1}{T} \int_{0}^{T} f_{n l}\left(\zeta_{X}, \dot{\zeta}_{X}, t\right) d t
$$

In the absence of non-linear terms, (5) simplifies into the usual linear frequency-domain formulation [2].

\section{B. Resolution Method}

(5) is a non-linear vector equation, which can be solved through a gradient-based method such as the Newton procedure, until $\|M X-F(X)-E\|^{2}$ is smaller that some given threshold $\nu$. Details about the resolution method and the gradient computation can be found in [5].

Besides the application domain, there are two significant differences between this work and that presented in [5]:

1) Firstly, the authors of [5] resort to statistical cubicization of the non-linear forces at each step of the Newton method, which enables them to compute the gradients through analytical formulae instead of requiring $2 N$ FFTs. Cubicization could also be useful for wave energy applications and deserves to be explored in future work, although such an approximation of the nonlinear forces would imply cost in accuracy.

2) Furthermore, unlike (1), the dynamical equations in [5] do not include any linear memory terms. As mentioned in II-A, using Ogilvie's relation [9], linear memory terms simplify easily through (5) and (6) when projecting (2) onto the basis of trigonometric functions.

The choice of an appropriate initial solution $X_{0}$ is crucial for the fast convergence of the NLFD method. The linear solution, obtained by setting $F(X)=0$ and solving the matrix inversion problem, $M X=E$, can provide a satisfactory starting point for the algorithm, although additional insight into the true solution may help in finding a more refined initial guess.

The computation of the Jacobian of the non-linear term, $F(X)$, at each iteration, necessitates $2 N$ fast Fourier transforms (FFTs), which results in a computational time increasing with the problem size in $N^{2} \ln (N)$. Therefore, for a given total simulated time, many, relatively short simulations should be preferred to fewer, longer simulations. However, some precaution should be taken on calibrating the problem size for each simulation:

1) If the frequency step $\Delta f=\frac{\omega_{0}}{2 \pi}=1 / T$ is too small, the computational time of the NLFD method increases, and makes it less competitive with respect to time-domain integration. In contrast, if $\Delta f$ is too large, the input and output spectra are poorly represented.

2) The maximum frequency that can be modelled is $f_{\max }=$ $N \Delta f=1 / 2 \Delta t$, and must be carefully chosen, depending on the typical speed of the device dynamics, and on the high-frequency content of the input wave spectrum.

\section{Comparison With Other Simulation Methods}

\section{A. Relationship With Time-Domain Methods}

1) Nature of the Output: The final outcome of the NLFD method, for a given signal, can be given both as a set of frequency-domain coefficients, or as a time-domain vector showing the periodic trajectory followed by a variable of interest. By running the NLFD method a few times only, with wave components randomly generated from the same wave spectrum, the spectral density function (SDF) of any output can be quickly and accurately computed, and time-domain statistics, such as probabilities of exceedance or zero-crossing rates, can be readily estimated. In contrast to TD integration, the NLFD formulation only allows for the consideration of steady state regimes.

2) Accuracy and Computational Time: If the system input can be modelled through a finite number of non-zero wave components, and if the steady-state response of the actual system is itself a continuously-differentiable, periodic, output, there is no theoretical limitation to the accuracy of the NLFD method, provided that $N$ is chosen sufficiently large. In particular, the radiation forces are used directly in their frequency-domain form, without any approximation. Then, an arbitrary accuracy can be achieved, by setting $\nu$ to a sufficiently small value. In addition, given the quadratic convergence rate of the Newton method [10], once the residuals are small enough, just one additional iteration can improve the accuracy by several orders of magnitude, while having little impact on the overall computation time.

In TD integration, the main computational burden consists of computing the radiation forces, which appear as a convolution integral in Cummins' equation (1). A direct computation of the integral makes it preferable to use numerical schemes with a fixed time step (see [1], Ch. 3). However, to circumvent the direct integral computation, various methods have been proposed in the literature [11], which approximate the radiation response function in the frequency or time domain, including a statespace approximation and Prony's method [4]. Such methods eliminate the need for calculating the convolution integral, and the system dynamics can be expressed as an ordinary first-order, state-space differential equation, where the number of states depends on the order of the radiation approximation. Within this scope, more efficient integration schemes, with adaptive time steps, can be easily adopted, such as the Dormand-Prince solver, which is the default method in the Matlab/Simulink simulation environment, and is widespread in the context of WEC simulation [1]. It is estimated that state-space or Prony's methods allow for dividing the computational time by a factor between 8 and 80 [4]. Nevertheless, the overall modelling accuracy is sensitive to the quality of the radiation approximation [4], while, with direct convolution and a constant time step method, the accuracy is guaranteed when the time step tends to zero [11].

In order to assess the accuracy of the NLFD method, in Section V a comparison is carried out against TD simulations, using a direct computation of the radiation convolution integral (and thus using a constant integration step). More precisely, an 
RK2 (or Improved Euler) scheme is chosen, which, amongst fixed-step methods, seems to be an interesting compromise in terms of computational time vs accuracy [11]. In this way, in spite of the technique simplicity, accuracy is guaranteed when the time step tends to zero [11], and thus a robust point of comparison is provided.

Radiation approximation methods are beyond the scope of the present study. Compared to RK2, such methods, combined with an adaptive-step solver, could provide a better trade-off between computational time and accuracy.

3) Range of Applicability: As far as the range of applicability is concerned, the NLFD formulation may show its limitations when dealing with systems whose outputs exhibit sharp edges or discontinuities for, at least, two reasons:

1) Motions featuring sharp edges could necessitate an excessive number $N$ of harmonic components in order to be accurately approximated. Thus, the computational cost of each Newton iteration in the NLFD method, which is proportional to $N^{2} \ln (N)$, could make the method inappropriate.

2) Explicit computation of the Jacobian, in the Newton method, implies that the non-linear functions are differentiable in the system variables. Hence, some discontinuous, highly non-linear forces may require a numerical estimation of the gradient, or alternatively an analytical, continuously-derivable approximation of the non-linear forces. In such a case, the accuracy of the NLFD method would be affected.

Well-known examples of such pathological cases are systems subject to Coulomb friction, e.g. some WECs equipped with a hydraulic PTO [12].

\section{B. Relationship With Spectral-Domain Linearisation}

1) The SD Method: The SD formulation has shown its ability to compute fast power estimates for WECs exhibiting weak, velocity-dependent non-linearities [1]. In its current implementations, the SD method operates under the assumption that both inputs and outputs can be modelled as stationary Gaussian processes. The method iterates in order to find the output SDF, say $S_{\zeta}(\omega)$. Although SD could also allow for statistical quadratisation or cubicisation [5], to date only linearisation has been applied to WEC modelling [1], while quadratisation and cubicisation involve significantly larger computational effort [5]. The following paragraphs explain how the SD linearisation procedure approximates the non-linear forces as a linear function of the velocity, and show that it cannot handle static non-linear forces.

a) Velocity-dependent non-linear forces: Let us consider a non-linear force of the form $f_{n l}=h(\dot{\zeta})$. The procedure presented in Chapter 4 of [1] requires the computation of the average power of the non-linear forces,

$$
P_{f_{n l}}=\langle h(\dot{\zeta}) \dot{\zeta}\rangle
$$

which, denoting $\delta v_{i}$ the infinitesimal velocity components of $\dot{\zeta}$, is then approximated as

$$
P_{f_{n l}} \approx \sum_{i}\left\langle\frac{\partial h}{\partial v_{i}}\right\rangle\left\langle\delta v_{i}^{2}\right\rangle=\left\langle h^{\prime}(\dot{\zeta})\right\rangle\left\langle\dot{\zeta}^{2}\right\rangle
$$

As a consequence, throughout the SD procedure, $h(\dot{\zeta})$ is approximated as a linear function of the form $\alpha \dot{\zeta}$, where $\alpha=\left\langle h^{\prime}(\dot{\zeta})\right\rangle$, given the current solution $\operatorname{SDF} S_{\zeta}$.

b) Static non-linear forces: Let us now consider a nonlinear force of the form $f_{n l}=g(\zeta)$. This time, the average power of the non-linear force is

$$
P_{f_{n l}}=\langle g(\zeta) \dot{\zeta}\rangle
$$

Note that $\zeta$ being a Gaussian process ensures independence of the random variables $\zeta$ and $\dot{\zeta}$ [13], which implies

$$
P_{f_{n l}}=\langle g(\zeta)\rangle\langle\dot{\zeta}\rangle=0
$$

Since $\dot{\zeta}$ is zero-mean. Thus, the static non-linear forces have no influence on the solution, so that the SD linearisation cannot take them into account. This is a significant limitation, since non-linear static terms, such as non-linear hydrostatic restoring forces, may have an influence on the relative phase of input and output wave components and, thus, on power production.

c) Mixed non-linear forces: Let us finally consider a mixed non-linear force of the form $f_{n l}=g(\zeta) h(\dot{\zeta})$, as in [14]. The statistical linearisation procedure results in computing the average power of the non-linear forces, as:

$$
P_{f_{n l}}=\langle g(\zeta) h(\dot{\zeta}) \dot{\zeta}\rangle
$$

which, using again the independence of $\zeta$ and $\dot{\zeta}$, and with the same linear approximation as in (8), results in

$$
P_{f_{n l}}=\langle g(\zeta)\rangle\langle h(\dot{\zeta}) \dot{\zeta}\rangle \approx\langle g(\zeta)\rangle\left\langle h^{\prime}(\dot{\zeta})\right\rangle\left\langle\dot{\zeta}^{2}\right\rangle
$$

Hence, again, the nonlinear force is ultimately approximated as a linear function of the form $\alpha \dot{\zeta}$, where $\alpha=\langle g(\zeta)\rangle\left\langle h^{\prime}(\dot{\zeta})\right\rangle$ for the solution $S_{\zeta}$.

2) Conclusions: A few remarks may now be formulated. Firstly, for the same frequency discretisation, the number of variables in the spectral-domain formulation is only half the number in the NLFD formulation (the $N$ amplitudes of $S_{\zeta}(\omega)$, and no phase information). Additionally, no demanding gradient computation is necessary for the SD method to work. Hence, the SD linearisation is certainly faster and more suitable than NLFD, provided that

1) only spectral-domain output statistics, such as average power or SDF, are of interest;

2) the significant non-linear forces are velocity-dependent;

3) the outputs are approximately Gaussian, and the nonlinearities can be reasonably approximated through a linear function of the form $\alpha \dot{\zeta}$ around the solution SDF, without compromising the accuracy of power estimates;

4) $\langle g(\zeta)\rangle$ and $\left\langle h^{\prime}(\dot{\zeta})\right\rangle$ can be easily derived from the current solution $\mathrm{SDF} S_{\zeta}$, which is always the case in the situations considered to date in WEC modelling [1], but may not always be true, depending on the functions $g$ and $h$.

The restrictions underpinning the SD procedure are more demanding than the assumptions necessary for the NLFD method, which are simply that the WEC response to a smooth, periodic signal can be well described as a truncated sum of sinusoids. In particular:

1) Unlike SD, the NLFD formulation can handle pure sinusoidal inputs, by simply filling other input components 
with zeros. Because of the non-linearities, the output components at other frequencies can be non-zero.

2) It may be possible that, for some non-linearities, the phases of the output sinusoidal components are not uniformly distributed - which would mean that the output is non-Gaussian. Unlike SD linearisation, the NLFD method allows for the study of such non-Gaussian outputs.

\section{CASE Studies}

The two WEC models considered, although relatively simple, are specifically chosen so as to highlight the characteristics of the NLFD method, as compared to the TD and SD formulations.

\section{A. Flap-Type WEC With Quadratic Viscous Drag Term}

The first case considered consists of a flap-type WEC, subject to a vortex shedding effect modelled by a quadratic viscous drag term of the form $f_{v}(\dot{\zeta})=-C_{v} \dot{\zeta}|\dot{\zeta}|$, where $\zeta$ denotes the angular position of the flap. The other hydrodynamic forces are modelled linearly, as well as the PTO which is represented as a simple linear damper, $f_{P T O}=-B_{P T O} \dot{\zeta}$.

Three variants of the model are studied:

1) a linear model, where $C_{v}$ is set to zero;

2) the baseline non-linear model, in which the viscous damping coefficient $C_{v}$ is given as in [7];

3) an intensified non-linear model, where the baseline drag coefficient is increased by $50 \%$.

\section{B. Heaving Sphere With Nonlinear Restoring Force and PTO}

The second test case consists of a spherical heaving point absorber with radius $R=2.5 \mathrm{~m}$, and aims at showing the suitability of the NLFD method to model highly-non-linear, positiondependent effects, and for studying parametric control design of the form $f_{P T O}:=u_{p}(\zeta, \dot{\zeta}, t)$, where $p$ is a set of parameters.

$\zeta$ denotes the displacement of the buoy gravity centre, relative to the free surface at rest. The buoy density is chosen to be half that of sea water, so that, at rest, $\zeta=0$. While radiation and excitation forces are modelled linearly, the hydrostatic restoring force is computed non-linearly, taking into account the immersed volume in the absence of waves:

$$
f_{h s}^{*}(\zeta)= \begin{cases}\pi \rho g\left(\frac{1}{3} \zeta^{3}-R^{2} \zeta\right) & \zeta \in[-R ; R] \\ -\frac{2}{3} \pi \rho g R^{3} & \zeta \geq R \\ \frac{2}{3} \pi \rho g R^{3} & \zeta \leq-R\end{cases}
$$

A viscous drag term is also taken into account, in the form $f_{v}(\dot{\zeta})=-C_{v} \dot{\zeta}|\dot{\zeta}|$.

Inspired by complex-conjugate control, which is optimal for monochromatic sea states, the parametric PTO force can be expressed as

$$
u_{B, K}(\zeta, \dot{\zeta})=-B \dot{\zeta}-K \zeta
$$

Given the natural dynamics of a relatively small heaving point absorber, which are significantly faster than common ocean wave periods, the appropriate setting of $K$, to maximise power production, is negative, so that the natural period of the controlled WEC is brought closer to typical wave periods.

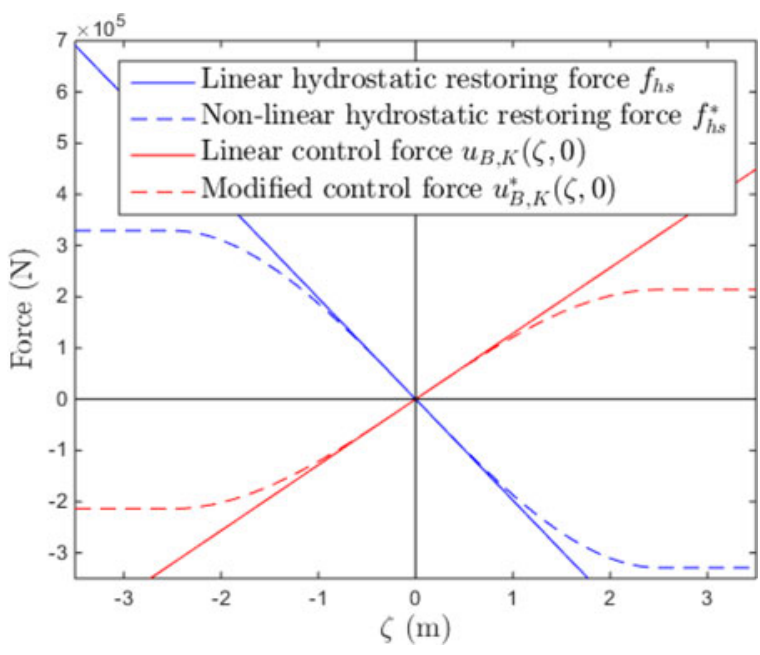

Fig. 1. Linear and non-linear restoring force and control force models (the latter taken at $\dot{\zeta}=0$ ).

However, with the hydrostatic restoring force being non-linearly computed, for large excursions of $\zeta$, the hydrostatic force $f_{h s}^{*}$ goes to a constant value while $u_{B, K}$ keeps increasing with $\zeta$ (see Fig. 1), thus pushing the device away from the free-surface, and allowing $\zeta$ and $u_{B, K}$ to tend to infinity.

In order to avoid such an unrealistic scenario, it is relevant to limit the control force magnitude, by replacing the term $-K \zeta$ with a function of the same form as (13), saturating in $\zeta=R$, so that:

$$
u_{B, K}^{*}(\zeta, \dot{\zeta})=-B \dot{\zeta}-\frac{K}{\pi \rho g R^{2}} f_{h s}^{*}(\zeta)
$$

As illustrated in Fig. 1, the appropriate stiffness properties in $-K \zeta$ are preserved around $\zeta=0$, while improving the physical significance of the WEC motion and PTO force.

In the case study, a wide range of $(K, B)$ pairs is explored, for a given sea state, in order to estimate the best possible combination in terms of power production.

\section{NUMERICAL RESULTS}

\section{A. Flap-Type WEC With Viscous Drag}

The flap-type WEC is a relevant point of comparison between TD, SD and NLFD methods, since it is a model for which the three formulations are suitable. The WEC is simulated in waves generated from a JONSWAP spectrum [15] with $H_{s}=$ $4 \mathrm{~m}$ and $T_{p}=16 \mathrm{~s}$. The period of the signal is $T=100 \mathrm{~s}$, and the frequency range is discretised into $N=50$ components.

For each of the three WEC model variants (linear, non-linear and intensified non-linear), 60 simulations are run, consisting of all possible combinations of 6 different PTO damping values, and 10 sets of random phases. Both NLFD and TD simulations are run to compute the WEC steady-state response in all the $3 \times 60=180$ cases. As far as SD is concerned, since the method is blind to the choice of input phases, only one run is necessary to obtain the full output characterisation - including average power output - for a given input wave spectrum and a given WEC model. Therefore, only one SD run per PTO value and 
WEC model variant is performed, i.e. $3 \times 6=18$ runs in total. In contrast, for TD and NLFD models, the use of 10 different sets of random phases means that, for each PTO value, the WEC is simulated in a total duration of $100 \times 10=1000 \mathrm{~s}$ to obtain a power estimate.

For the TD simulations, a $50 \mathrm{~s}$ transient time was added at the beginning of each simulation, simply by concatenating the last $50 \mathrm{~s}$ of the periodic $100 \mathrm{~s}$ signal at the beginning of the simulation window. Of course, for average power computation and comparison with the two other methods, only the $100 \mathrm{~s}$ of steady-state motion are considered. As mentioned in Section III-A, the TD numerical integration scheme is a simple RK2, for which the influence of the time step length on the results accuracy is examined. RK2 results are then presented for 6 different time-step values, ranging from 0.05 to $0.001 \mathrm{~s}$. The convolution product for radiation forces is directly computed, with a truncation of the integral to a range of $20 \mathrm{~s}$.

Finally, let us note that, for the linear model, both SD and NLFD methods reduce to the classical, linear frequency-domain method, and thus are strictly equivalent and do not necessitate any iteration after the linear initialisation.

1) Comparison Between RK2 and NLFD Results: For each of the 3 models and 60 simulation conditions, both RK2 and NLFD are used to solve the steady-state equations of motion. Three main features are considered for comparison:

1) The $\%$ power production difference, which quantifies the agreement between the average power values obtained using the different methods, computed as

$$
\epsilon_{\% P}=100 \times \frac{P_{\mathrm{TD}}-P_{\mathrm{NLFD}}}{P_{\mathrm{NLFD}}}
$$

2) The \% root-mean-square (RMS) difference, which measures the agreement between the WEC velocity patterns obtained using RK2 and NLFD methods, computed as

$$
\epsilon_{\% R M S}=100 \times \sqrt{\frac{\int_{0}^{T}\left(\dot{\zeta}_{\mathrm{TD}}(t)-\dot{\zeta}_{\mathrm{NLFD}}(t)\right)^{2} d t}{\int_{0}^{T} \dot{\zeta}_{\mathrm{NLFD}}(t)^{2} d t}}
$$

3) The computational time per simulation, $t_{\mathrm{CPU}}$, normalised by the NLFD computational time.

The average values for $\epsilon_{\% R M S}$ and $\epsilon_{\% P}$ over the 60 simulations are summarised in Fig. 2(a) and (b) for the three models.

Regardless of the model, Fig. 2(a) shows that the \% difference in power estimates decreases proportionally with the RK2 timestep, so that $P_{\mathrm{NLFD}}$ appears to be the limit of $P_{\mathrm{TD}}$ when the time-step tends to zero. Accordingly, Fig. 2(b) indicates that the periodic, steady-state velocity pattern obtained through RK2 simulations, is increasingly close to the NLFD velocity pattern, even though a negligible \% RMS difference seems to remain, even when the time step tends to zero.

The results of Fig. 2(a) and (b) can be more easily understood when considering the linear model. Indeed, given that the input is a finite sum of harmonic sinusoids, the actual steadystate response of the linear system can be exactly computed, through multiplication of the input by the linear transfer function. Thus the NLFD result is exact. In contrast, TD integration

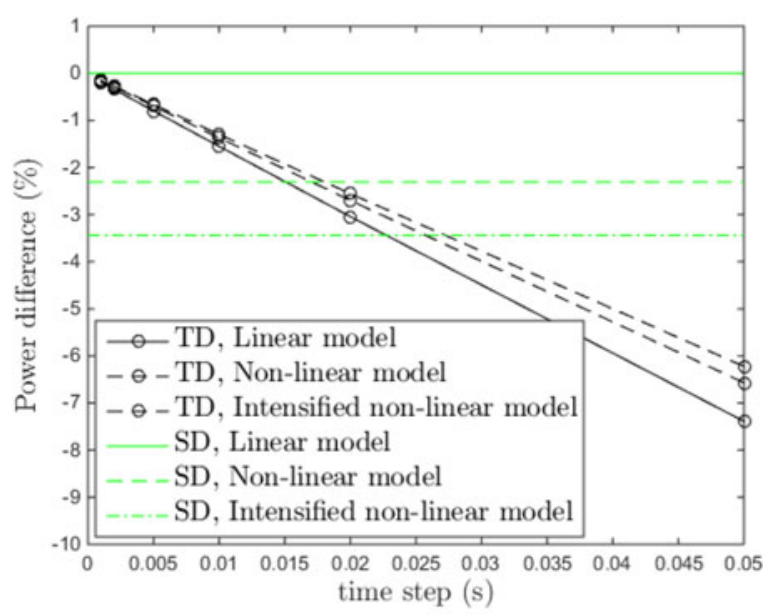

(a)

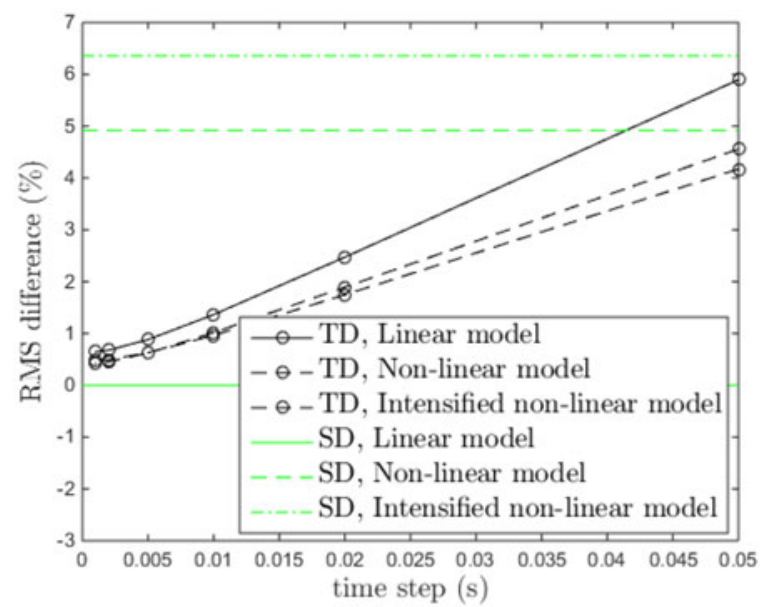

(b)

Fig. 2. Comparison of TD and SD results with NLFD (a) Average power difference between TD (resp. SD) and NLFD results (expressed in \% of the NLFD results) (b) Average \% RMS difference between TD (resp. SD) and NLFD time-domain velocity profiles.

only results in an approximation of the true response. It is then obvious that the TD integration response comes closer to the actual steady-state response as the RK2 time step approaches zero.

Non-linear and intensified non-linear model results are consistent with those obtained from the linear model. Indeed, as mentioned previously in Section III-A, for $N$ chosen sufficiently large, any arbitrary accuracy $\nu$ can be reached without significantly affecting the computational time. So, even though, in contrast to the linear case, the NLFD method does not give the exact solution, it can find an approximation with a level of accuracy not attainable through RK2 integration with reasonable time steps.

For the baseline non-linear model, Fig. 3 confirms that the results suggested in Fig. 2(a) and (b) are consistent over the range of PTO values investigated. Also based exclusively on the baseline non-linear model, Table I summarises the results in terms of accuracy and computational time.

As far as computational efficiency is concerned, it can be seen from Table I that the NLFD method is significantly faster 


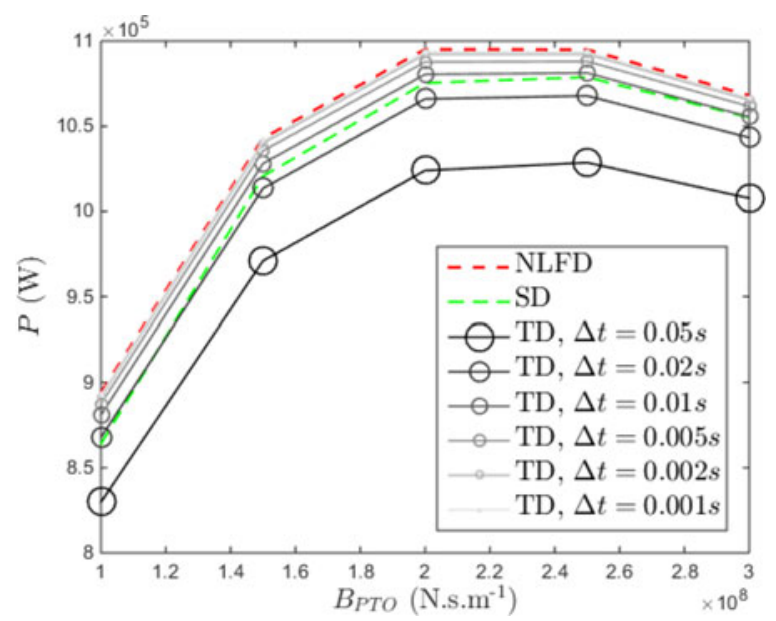

Fig. 3. Average WEC power, with varying PTO coefficient and simulation methods (baseline model).

TABLE I

Comparative TABLE of TD (RK2), SD AND NLFD Methods, APPLIED to THE FLAP-TYPe WEC MODEL ( $t_{C P U}$ IS NORMALISED)

\begin{tabular}{lcccccccc}
\hline \hline \multicolumn{1}{c}{$\mathrm{TD}(\mathrm{RK} 2)$} & & & NLFD & $\mathrm{SD}$ \\
\hline$\Delta t$ & 0.001 & 0.002 & 0.005 & 0.01 & 0.02 & 0.05 & & \\
$\epsilon_{\% \text { RMS }}$ & 0.43 & 0.45 & 0.62 & 1.0 & 1.9 & 4.6 & 0 & 5.17 \\
$\epsilon_{\%} P$ & -0.16 & -0.29 & -0.7 & -1.4 & -2.7 & -6.6 & 0 & -1.66 \\
$t_{\mathrm{CPU}}$ & 13600 & 4200 & 1200 & 310 & 117 & 35 & 1 & 0.4 \\
Sim./Spec & $\sim 5$ & $\sim 5$ & $\sim 5$ & $\sim 5$ & $\sim 5$ & $\sim 5$ & $\sim 5$ & 1 \\
\hline \hline
\end{tabular}

than any of the RK2 methods considered. In particular, to obtain the correct power value within a $1 \%$ accuracy, RK2 requires a $0.005 \mathrm{~s}$ time step and is approximately 1000 times slower than the NLFD procedure.

2) Comparison Between SD and NLFD Results: Unlike TD and NLFD formulations, SD only necessitates one run of the method per input sea state - for given WEC parameters. The SD method was found to be (see Table I) about 3 times faster than the NLFD method for each run but, considering that approximately 5 NLFD (or TD) runs are necessary to obtain an accurate estimate of the output power spectrum, the SD method can be viewed as being about 15 times faster than the NLFD method.

For power production assessment, looking at Figs. 2(a) and 3 and Table I, one can see that the SD method has an accuracy comparable to, for example, the TD method with a $0.01 \mathrm{~s}$ time step, but a computational time smaller than the NLFD method.

However, as mentioned in Section III-B, the SD method is less suitable for the study of output signals in the time-domain. To illustrate this point, for each given PTO value and WEC model, the final value of the linear coefficient $\alpha$ for the approximation of the non-linear forces was saved. Then, for each of the 10 sets of random phases, it was applied to compute the time-domain output as a linear function of the wave input. The resulting timedomain velocity trajectory was finally compared to the NLFD outputs, using $\epsilon \% R M S$ similarly to (17). Fig. 2(b) shows that, in terms of $\epsilon_{\% R M S}$, SD performs worse than TD integration
TABLE II

Comparative TABle of TD (RK2), SD AND NLFD Methods, APPLIED TO THE HEAVING SPHERE MODEL ( $t_{C P U}$ IS NORMALISED)

\begin{tabular}{lccccc}
\hline \hline \multicolumn{5}{c}{$\mathrm{TD}(\mathrm{RK} 2)$} & NLFD \\
\hline$\Delta t$ & 0.005 & 0.01 & 0.02 & 0.05 & \\
$\epsilon_{\% R M S}$ & 0.23 & 0.25 & 0.32 & 0.62 & 0 \\
$\epsilon_{\% P}$ & -0.12 & -0.21 & -0.39 & -0.99 & 0 \\
$t_{\mathrm{CPU}}$ & 2000 & 157 & 61 & 20 & 1 \\
\hline \hline
\end{tabular}

using the coarser time step studied (for the non-linear models, of course).

\section{B. Heaving Sphere With Non-Linear Restoring Force and PTO}

Unlike the flap with viscous drag, the heaving-sphere case contains static non-linear forces, which cannot be handled through the SD formulation. Thus only TD and NLFD formulations are compared here. The WEC is simulated in waves generated from a JONSWAP spectrum [15] with $H_{s}=3 \mathrm{~m}$ and $T_{p}=9 \mathrm{~s}$. The period of the signal is $T=60 \mathrm{~s}$, and the frequency range is discretised into $N=50$ components. A linear model, using the linearised restoring force $f_{h s}$ and linear control force $u_{B, K}$, is used for initialisation.

A wide range of $\left(B_{p t o}, K_{P T O}\right)$ pairs are investigated, consisting of 17 values for $B_{P T O}$ and 10 values for $K_{P T O}$, for a total of 170 combinations. The periodic input signal is generated from 10 different sets of random phases; thus the total number of simulations is 1700, in which both NLFD and TD are run to compute the WEC steady-state response (then, for each PTO setting, the total simulated duration is $10 \times 60=600 \mathrm{~s}$ ). Given the large number of simulations involved, RK2 integration with the 0.001 and $0.002 \mathrm{~s}$ time steps is intractable within a reasonable amount of time; therefore RK2 results are only presented for time steps from 0.005 to 0.05 .

The comparison between TD and NLFD results relies on the same three metrics as for the flap case, namely $\epsilon_{\% R M S}$ (17), $\epsilon_{0 P}$ (16) and $t_{C P U}$, whose averages over the 1700 simulations are summarised in Table II.

The results shown in Figs. 4(a), (b), and 5 are very similar to those presented for the flap in Section V-A: the difference between NLFD and TD results, in terms of average power estimate and velocity profile, decreases with the RK2 time step, even though a negligible difference between the two velocity patterns seems to remain, even when the RK2 time step tends to zero.

It can be noticed that the magnitude of $\epsilon_{\% R M S}$ and $\epsilon_{\% P}$ is significantly smaller than in the flap case (less than $1 \%$ vs more than 5\%), which means that RK2 results are more accurate in the sphere case than in the flap case, for a given time step. A probable explanation lies with the radiation forces which, as highlighted in Section V-A, are a major source of approximation for TD methods, and whose relative influence is significantly more important for the flap modelled considered, than for the sphere model.

The difference between NLFD and RK2 results with decreasing time steps were found to be consistent across the range of 


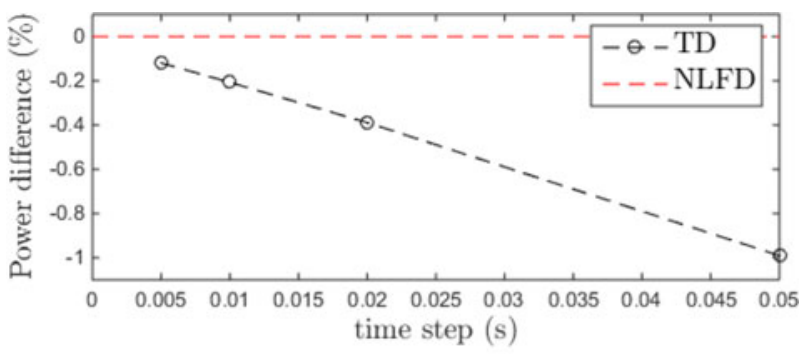

(a)

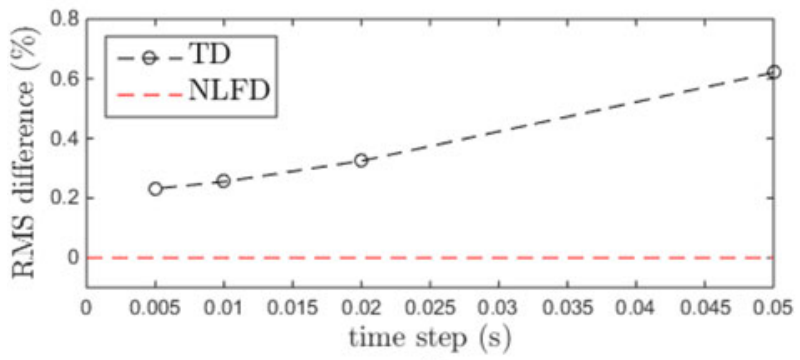

(b)

Fig. 4. TD results with different time steps compared with NLFD results (a) Average power difference between TD and NLFD results (expressed in \% of the NLFD results). (b) Average \% RMS difference between TD and NLFD time-domain velocity profiles.



Fig. 5. Average WEC power, with varying PTO coefficient and simulation methods $\left(K_{P T O}=-1.4 \times 10^{5} \mathrm{~N} \cdot \mathrm{m}^{-1}\right)$.

conditions investigated, as illustrated in Fig. 5 for a fixed $K_{P T O}$ value and a small range of $B_{P T O}$ parameters.

In terms of computational time, NLFD was found, again, to be significantly faster than any of the RK 2 methods considered (see Table II). In practice, using a $3.50 \mathrm{GHz}, 8$-core Intel processor, the average computational time for the NLFD method was $0.0036 \mathrm{~s}$, so that the 1700 simulations could be run in just 6 s - while several hours were necessary for the 1700 RK2 simulations with a time step of $0.005 \mathrm{~s}$.

Finally, in order to illustrate the applicability of the NLFD method to parametric optimisation, Fig. 6 shows the power output results over the range of $\left(B_{P T O}, K_{P T O}\right)$ values considered (using NLFD). A well-defined maximum can be seen, and suggests that, using the parametric control introduced in IV-B with optimal settings, the power output can be multiplied by more

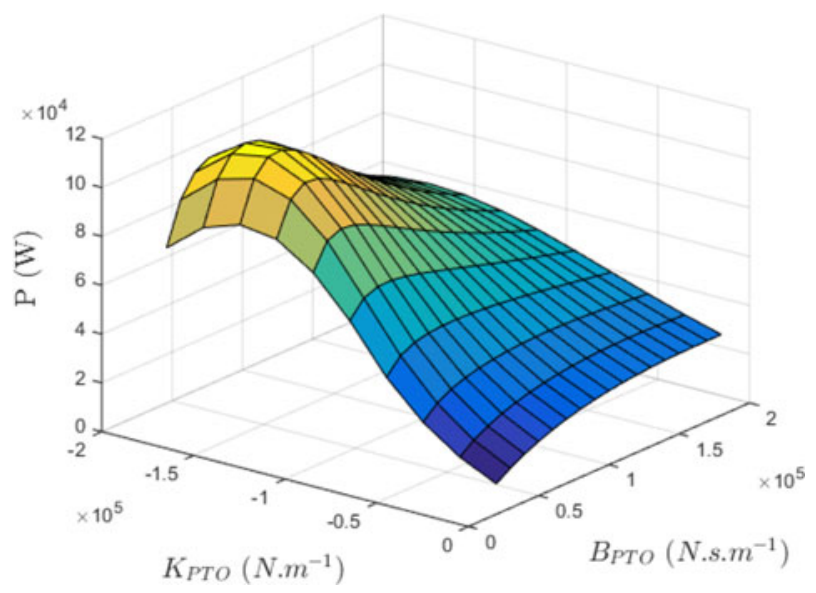

Fig. 6. Average WEC power, with varying PTO damping and stiffness values.

than three with respect to the best passive control (i.e. with $\left.K_{P T O}=0\right)$.

\section{DISCUSSION}

\section{A. Benefits of the Method}

The numerical results obtained in $\mathrm{V}$ show that the NLFD method can be successfully applied to WEC simulation with strong non-linearities.

At little additional computational expense, NLFD can overcome the limitations inherent to SD linearisation, both in terms of accuracy and applicability domain. However, for some applications where SD is particularly relevant, such as power production estimates for the flap-type WEC considered in this study, $\mathrm{SD}$ is significantly faster than NLFD, for a negligible cost in accuracy.

The computational efficiency and accuracy of the NLFD method compare favourably to RK2 integration. More precisely, with adequate settings, i.e. with a large enough number of harmonics $N$ and a small enough convergence threshold $\nu$, NLFD results approach the limit of constant time-step TD results, when the time step tends to zero. NLFD accuracy seems particularly advantageous when the linear radiation forces play a prominent role in the WEC dynamics.

With regard to computation time, the relative performance of TD and NLFD, such as presented in Section V, would change depending on the chosen TD numerical integration scheme. However, any significant improvement in the TD computational time would require an adaptive step solver, which would involve the use of an approximation technique for the radiation forces, as explained in Section III-A. A detailed comparison of NLFD and TD computational properties, using radiation approximation techniques for TD integration, could be the subject of future work, to assess more robustly the benefits of NLFD, in terms of trade-off between computational time and accuracy. In addition to radiation approximation techniques, the use of a variable step solver, combined with a direct computation of the integral using interpolation, could be included in the comparison. 
Nevertheless, the NLFD method elegantly deals with linear radiation forces, without resorting to any approximation, and therefore can already be seen as an interesting alternative to radiation force approximation methods: the reduction in computational time seems to be in the same order of magnitude, without any loss of accuracy.

Combining computational efficiency with physical insight, NLFD could be a powerful tool for the applications mentioned in Section I, which require a large number of WEC simulations, such as parametric design or control optimisation, power assessment, or a probabilistic study of WEC outputs.

\section{B. Limitations and Further Developments}

However, NLFD is subject to specific limitations. Firstly, as explained in Section III-A in terms of range of applicability, NLFD necessitates the existence of an appropriate analytical WEC model, preferably involving differentiable functions for easy use and fast convergence of Newton's method. In particular, NLFD may not be appropriate when the solution for the dynamical equations is non-smooth (i.e. with discontinuous derivatives).

Furthermore, the convergence of the Newton method cannot always be ensured, depending on the algorithm starting point and on the nature of the non-linear functions, although none of the cases presented in this study was subject to such an issue. If needed, more refined initialisation methods and, above all, more sophisticated versions of the Newton procedure [10], could be considered, perhaps at the expense of the convergence rate.

For both WEC models studied here, the number of frequency components has been set, somewhat arbitrarily, to $N=50$, which, in both cases, corresponds to a signal period $T$ approximately equal to 6 peak wave periods. For specific applications of the NLFD method, the joint influence of $N$ and $T$ on power production estimates would deserve to be investigated.

Advantages of the NLFD method extend to inclusion of parametric control such as in Sections IV-B and V-B, but cannot model the effect of a sophisticated real-time control loop, for example using a receding-horizon perspective [16], especially when constraints must be included.

As explained in [5], quadratisation and cubicisation of the non-linear forces can also be carried out within the NLFD framework, enabling significant computational savings in the calculation of the Jacobian for the Newton method.

Finally, although the NLFD formulation here includes only one DoF, the extension of the method to more DoFs doesn't pose any theoretical issue [6], and could be the subject of future work for WECs with multiple DoFs.

\section{REFERENCES}

[1] M. Folley, Numerical Modelling of Wave Energy Converters. New York, NY, USA: Academic, 2016.

[2] J. Falnes, Ocean Waves and Oscillating Systems: Linear Interactions Including Wave-Energy Extraction. Cambridge, U.K: Cambridge Univ. Press, 2002.

[3] W. Cummins, "The impulse response function and ship motion," Schiffstechnik, vol. 47, no. 9, pp. 101-109, 1962.
[4] J. A. Armesto, R. Guanche, F. Del Jesus, A. Iturrioz, and I. J. Losada, "Comparative analysis of the methods to compute the radiation term in Cummins' equation," J. Ocean Eng. Mar. Energy, vol. 1, no. 4, pp. 377393, 2015.

[5] P. Spanos, M. Di Paola, and G. Failla, "A Galerkin approach for power spectrum determination of nonlinear oscillators," Meccanica, vol. 37, no. $1 / 2$, pp. $51-65,2002$.

[6] G. Failla, P. Spanos, and M. Di Paola, "Response power spectrum of multidegree-of-freedom nonlinear systems by a Galerkin technique," J. Appl. Mech., vol. 70, no. 5, pp. 708-714, 2003.

[7] G. Bacelli, "Optimal control of wave energy converters," Ph.D. dissertation, Elect. Eng. Dept., National Univ. Ireland Maynooth, Maynooth, Ireland, 2014.

[8] S. D. Conte and C. W. De Boor, Elementary Numerical Analysis: An Algorithmic Approach. Columbus, OH, USA: McGraw-Hill, 1980.

[9] T. Ogilvie, "Recent progress toward the understanding and prediction of ship motions," in Proc. 5th Symp. Naval Hydrodyn., Washington DC, 1964, pp. 3-12.

[10] M. S. Bazaraa, H. D. Sherali, and C. M. Shetty, Nonlinear Programming: Theory and Algorithms. Hoboken, NJ, USA: Wiley, 2013.

[11] A. Kurniawan, J. Hals, and T. Moan, "Assessment of time-domain models of wave energy conversion systems," in Proc. 9th Eur. Wave Tidal Energy Conf., Univ. Southampton, Southampton, U.K., 2011.

[12] A. Falcão, "Modelling and control of oscillating-body wave energy converters with hydraulic power take-off and gas accumulator," Ocean Eng., vol. 34, no. 14, pp. 2021-2032, 2007.

[13] M. K. Ochi, Ocean Waves: The Stochastic Approach, vol. 6. Cambridge, U.K: Cambridge Univ. Press, 2005.

[14] M. Folley and T. Whittaker, "Spectral modelling of wave energy converters," Coastal Eng., vol. 57, no. 10, pp. 892-897, 2010.

[15] K. Hasselmann et al., "Measurements of wind-wave growth and swell decay during the Joint North Sea Wave Project (JONSWAP)," Ergaenzungsheft zur Deutschen Hydrographischen Zeitschrift, Reihe A(8), 12, p. $95,1973$.

[16] R. Genest and J. V. Ringwood, "Receding horizon pseudospectral control for energy maximization with application to wave energy devices," IEEE Trans. Control Syst. Technol., vol. 25, no. 1, pp. 29-38, Jan. 2017.

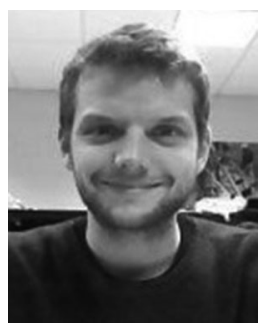

Alexis Mérigaud received the Engineering degree in 2012 from ENSTA, Paris, France, where he specialized in energy management and economics. He then studied Public administration for two years at Sciences Po Paris, Paris, France. Since January 2015 he has been working toward the Ph.D. degree at the Centre for Ocean Energy Research in Maynooth University, Maynooth, Ireland. He is working on energy forecasting and predictive maintenance applied to marine renewable energy sources. His previous experience in the field of energy includes economic modeling, as well as technical regulatory aspects, of energy networks and markets.

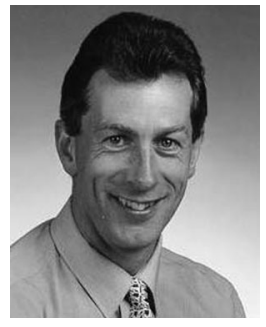

John Ringwood received the Diploma degree in electrical engineering from Dublin Institute of Technology, Dublin, Ireland, and the Ph.D. degree in control system from Strathclyde University, Glasgow, Scotland, in 1981 and 1985, respectively. He is currently a Professor of electronic engineering and the Director of the Centre for Ocean Energy Research, Maynooth University Ireland, Maynooth, Ireland. From 2000 to 2005, he was the Head of the Electronic Engineering Department at NUI Maynooth, developing the department from a greenfield site. His research interests include time series modeling, wave energy, control of plasma processes, and biomedical engineering. He is a Chartered Engineer and a Fellow of the Institution of Engineers of Ireland. 